\title{
Quantum field theoretical study of an effective spin model in coupled optical cavity arrays
}

\author{
Sujit Sarkar \\ Poornaprajna Institute of Scientific Research, \\ 4 Sadashivanagar, \\ Bangalore 5600 80, India. \\ E-Mail: sujit@physics.iisc.ernet.in \\ Phone: 09180 23612511/23619034, \\ Fax: 091-80-2360-0228
}

(Dated: January 5, 2019)

\begin{abstract}
Atoms trapped in microcavities and interacting through the exchange of virtual photons can model an anisotropic Heisenberg spin-1/2 lattice. We do the quantum field theoretical study of such a system using the Abelian bosonization method followed by the renormalization group analysis. We present interesting physics due to the presence of exchange anisotropy. An infinite order Kosterliz-Thouless-Berezinskii transition is replaced by second order XY transition even an infinitesimal a small anisotropy in exchange coupling is introduced. We predict a quantum phase transition between Mott insulating and photonic superfluid phase due to detuning between the cavity and laser frequency, a large detuning favours the photonic superfluid phase. We also do the analysis of Jaynes and Cumming Hamiltonian to support results of quantum field theoretical study.

Pacs: $42.50 . \mathrm{Dv}, 42.50 . \mathrm{Pq}$, 03.67.Bg, 75.10.Jm

Keywords:Quantum Many Body Models, Polariton, Cavity QED and Spin Chain Model
\end{abstract}




\section{INTRODUCTION}

The physics of strongly correlated system is interesting in its own right and manifests in different branches of physics. Some of the important correlated physics appears in natural oxide materials [1] and some of them are in engineered materials, like the correlated

physics in Josephson junction array [2], Bose-Einstein condensation and optical lattice [3, 4]. Therefore one can raise the question what is the further source of correlated physics in the state of enginnering ?. The recent experimental success in engineering strong interaction between the photons and atoms in high quality micro-cavities opens up the possibility to use light matter system as quantum simulators for many body physics [5, 6. 7, 8, 9, 10, 11, 12, 13, 14, 15, 16, 17].

Here we would like to discuss the basic physics of micro-optical cavity very briefly. A microcavity can be created in a photonic band gap material by producing a localised defect in the structure of the crystal, in such a way that light of a particular frequency can not propage outside the defect area. Large arrays of such micro-cavities have been produce. Photon hopping between neighboring cavities has been observed in the microwave and optical domains. Many body Hamiltonians can be created and probed in coupled cavity arrays. There atoms are used for detection and also for the generation of interaction between photons in the same cavity. As the distance between the adjacent cavities is considerably larger than the optical wave length of the resonant mode, individual cavities can be addressed. These artificial system could act as a quantum simulator. In this optical cavities system we study the different quantum phase of polariton ( a combined excitations of atom-photon interactions. ) by using spin models that conserve the total number of excitations. Best of our knowledge, at first we have done the explcit quantum field theoretical calculations of this type of system.

At first we would like to discuss the generation of the spin model for such type of systems. It has already been discussed in the literature but we mention this process very briefly for the sake of completeness. In this description we will follow the Ref. [6] and Ref. [7].

Micro-cavities of the photonic crystal are coupled through the exchange of photons. Each cavity consists of one atom with three levels in the energy spectrum, two of them are long lived and represents two spin states of the system and the other is excited. Externally applied laser and cavity modes couple to each atom of the cavity. It may induce the Raman 
transition between these two long lived levels. Under a suitable detuning between the laser and the cavity modes virtual photons are created in the cavity which mediate an interaction with another atom in a neighboring cavities. One can eliminated the excited states of atomic level photon states by choosing the appropriate detuning between the applid laser and cavity modes. Then one can achieve only two states per atom in the long lived state and the system can be described by a spin-1/2 Hamiltonian [6, 7].

Fig. 1 shows the schematic phase diagram of our description to generate the xy spin interaction of the system. The Hamiltonian of the system consists of three parts:

$$
H=H_{A}+H_{B}+H_{C}
$$

Hamiltonians are following

$$
H_{A}=\sum_{j=1}^{N} \omega_{e}\left|e_{j}><e_{j}\right|+\omega_{a b}\left|b_{j}><b_{j}\right|
$$

$j$ is the cavity index. $\omega_{a b}$ and $\omega_{e}$ is the energy of the state $\mid b>$ and the excited state respectively. The energy level of state $\mid a>$ is set zero. The following Hamiltonian describes photons in the cavity,

$$
H_{C}=\omega_{C} \sum_{j=1}^{N} a_{j}^{\dagger} a_{j}+J_{C} \sum_{j=1}^{N}\left(a_{j}^{\dagger} a_{j+1}+h . c\right),
$$

where $a_{j}^{\dagger}$ creates a photon in cavity $j, \omega_{C}$ is the energy of photons and $J_{C}$ is the tunneling rate of photons between neighboring cavities. Interaction between the atoms and photons and also by the driving lasers are described

$$
H_{A C}=\sum_{j=1}^{N}\left[\left(\frac{\Omega_{a}}{2} e^{-i \omega_{a} t}+g_{a} a_{j}\right)\left|e_{j}><a_{j}\right|+h . c\right]+[a \leftrightarrow b] .
$$

Here $g_{a}$ and $g_{b}$ are the couplings of the cavity mode for the transition from energy states $\mid a>$ and $\mid b>$ to the excited state. $\Omega_{a}$ and $\Omega_{b}$ are the Rabi frequencies of lasers with frequencies $\omega_{a}$ and $\omega_{b}$ respectively.

They have derived an effective spin model by considering the following physical processes: A virtual process regarding emission and absorption of photons between the two stable states of neghiboring cavities. The resulting effective Hamiltonian is

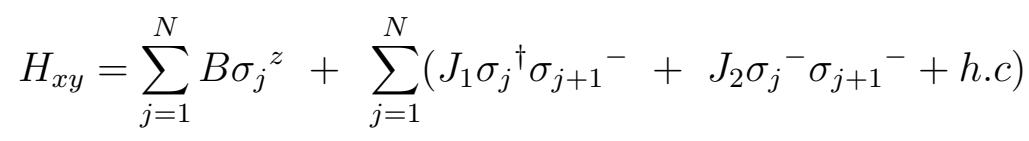


When $J_{2}$ is real then this Hamiltonian reduces to the XY model. Where $\sigma_{j}^{z}=\mid b_{j}><$ $b_{j}|-| a_{j}><a_{j}\left|, \sigma_{j}^{+}=\right| b_{j}><a_{j}\left|, \sigma_{j}{ }^{-}=\right| a_{j}><b_{j} \mid$

$$
H_{x y}=\sum_{i=1}^{N} B\left(\sigma_{i}^{z}+J_{x} \sigma_{i}^{x} \sigma_{i+1}^{x}+J_{y} \sigma_{i}^{y} \sigma_{i+1}^{y}\right) .
$$

With $J_{x}=\left(J_{1}+J_{2}\right) / 2$ and $J_{y}=\left(J_{1}-J_{2}\right) / 2$.

Here we discuss very briefly about an effective $\sigma_{i}{ }^{z} \sigma_{i}{ }^{z}$ in such a system. Authors of Ref.[6, 7] have proposed the same atomic level configuration but having only one laser of frequency $\omega$ that mediates atom-atom coupling through virtual photons. Another laser field with frequency $\nu$ is used to tune the effective magnetic field. They described the onedimensional case. In this case the Hamiltonian $H_{A C}$ will change but the Hamiltonians $H_{A}$ and $H_{C}$ will not.

$$
H_{A C}=\sum_{j=1}^{N}\left[\left(\frac{\Omega_{a}}{2} e^{-i \omega_{a} t}+\frac{\Lambda_{a}}{2} e^{-i \nu_{a} t} g_{a} a_{j}\right)\left|e_{j}><a_{j}\right|+h . c\right]+[a \leftrightarrow b] .
$$

Here, $\Omega_{a}$ and $\Omega_{b}$ are the Rabi frequencies of the driving laser with frequency $\omega$ on transition $|a>\rightarrow| e>|b>\rightarrow| e>$, whereas $\Lambda_{a}$ and $\Lambda_{b}$ driving laser with frequency $\nu$ on transition $|a>\rightarrow| e>|b>\rightarrow| e>$. They have eliminated adiabatically the excited atomic levels and photons by considering the interaction picture with respect to $H_{0}=H_{A}+H_{C}$. They have considered the detuning parameter in such a way that the Raman transitions between two level supressed. They have also chosen the parameter in such a way that the dominant two-photon processes are those that involve one laser photon and one cavity photon each but the atom does no transition between levels a and b. Whenever two atoms exchange a virtual photon then both of them experience a Stark shift plays the role of an efective $\sigma^{z} \sigma^{z}$ interaction. Then the effective Hamiltonian reduce to

$$
H_{z z}=\sum_{j=1}^{N}\left(\tilde{B}{\sigma_{j}}^{z}+J_{z}{\sigma_{j}}^{z} \sigma_{j+1}{ }^{z}\right)
$$

Analytical expressions for $\tilde{B}, J_{1}, J_{2}$ and $J_{z}$ has given in Ref. [7]. These two parameters can be tuned independently by varying the laser frequencies. They have obtained an effective model by combining Hamiltonians $H_{x y}$ and $H_{z z}$ by using Suzuki-Trotter formalism. The effective Hamiltonian simulated by this procedure is

$$
H_{\text {spin }}=\sum_{j=1}^{N}\left(B_{\text {tot }} \sigma_{j}{ }^{z}+\sum_{\alpha=x, y, z} J_{\alpha} \sigma_{j}{ }^{\alpha} \sigma_{j+1}{ }^{\alpha}\right)
$$


where $B_{t o t}=B+\tilde{B}$. It has been shown in Ref. [7] that $J_{y}$ is less than than $J_{x}$. It is clear from analytical expressions for $J_{x}$ and $J_{y}$ that the magnitudes of $J_{1}$ and $J_{2}$ are different. This result of numerical simulations trigger us to define a model, which has given below to study the quantum phases of this system and also the transition among them and at the same time this subject is in the state of art of engineering.

\section{RENORMALIZATION GROUP STUDY OF MODEL HAMILTONIAN}

We consider the anisotropic Heisenberg spin-1/2 Hamiltonian on a one dimensional lattice. The XYZ Heisenberg Hamiltonians is defined as:

$$
\begin{aligned}
& H_{X Y Z}=\sum_{n}\left[(1+a) S_{n}^{x} S_{n+1}^{x}+(1-a) S_{n}^{y} S_{n+1}^{y}\right. \\
& \left.+\Delta S_{n}^{z} S_{n+1}^{z}+h S_{n}^{z}\right],
\end{aligned}
$$

where $S_{n}^{\alpha}$ are the spin-1/2 operators. We assume that the $X Y$ anisotropy $a$ and the $z z$ coupling $\Delta$ satisfy $-1 \leq \Delta \leq 1$, and $0<a \leq 1$ and magnetic field strength $h \geq 0$. The Hamiltonian $H_{X Y Z}$ is invariant under the transformation $S_{n}^{x} \rightarrow-S_{n}^{x}, S_{n}^{y} \rightarrow-S_{n}^{y}, S_{n}^{z} \rightarrow-S_{n}^{z}$, actually it is a $Z_{2}$ symmetry. For finite $h, Z_{2}$ symmetry is absent when $S_{n}^{z} \rightarrow-S_{n}^{z}$. Here $h \sim B_{\text {tot }}, \Delta=J_{z}, J_{1}=1$ and $J_{2}=a$.

Spin operators can be recasted in terms of spinless fermions through Jordan-Wigner transformation and then finally one can express the spinless fermions in terms of bosonic fields 19]. We recast the spinless fermions operators in terms of field operators by this relation [19].

$$
\psi(x)=\left[e^{i k_{F} x} \psi_{R}(x)+e^{-i k_{F} x} \psi_{L}(x)\right]
$$

where $\psi_{R}(x)$ and $\psi_{L}(x)$ describe the second-quantized fields of right- and the left-moving fermions respectively. $k_{F}$ is Fermi wave vector. Therefore, one can study the effect of gate voltage through arbitrary $k_{F}$. We would like to express the fermionic fields in terms of bosonic field by the relation

$$
\psi_{r}(x)=\frac{U_{r}}{\sqrt{2 \pi \alpha}} e^{-i(r \phi(x)-\theta(x))}
$$

$r$ is denoting the chirality of the fermionic fields, right (1) or left movers $(-1)$. The operators $U_{r}$ commutes with the bosonic field. $U_{r}$ of different species commute and $U_{r}$ of the same 
species anti-commute. $\phi$ field corresponds to the quantum fluctuations (bosonic) of spin and $\theta$ is the dual field of $\phi$. They are related by the relations $\phi_{R}=\theta-\phi$ and $\phi_{L}=\theta+\phi$.

Hamiltonian $H_{0}$ is non-interacting part of $H_{X Y Z}$.

$$
H_{0}=\frac{v}{2} \int d x\left[\left(\partial_{x} \theta\right)^{2}+\left(\partial_{x} \phi\right)^{2}\right]
$$

where $v$ is the velocity of the low-energy excitations. It is one of the Luttinger liquid parameters and the other is $K$, which is related to $\Delta$ by [19]

$$
K=\frac{\pi}{\pi+2 \sin ^{-1}(\Delta)}
$$

$K$ takes the values 1 and $1 / 2$ for $\Delta=0$ (free field), and $\Delta=1$ (isotropic anti-ferromagnet), respectively. The relation between $K$ and $\Delta$ is not preserved under the renormalization, so this relation is only correct for initial Hamiltonian. The analytical form of the spin operators in terms of the bosonic fields are

$$
\begin{aligned}
S_{n}^{x} & =\left[c_{2} \cos (2 \sqrt{\pi K} \phi)+(-1)^{n} c_{3}\right] \cos \left(\sqrt{\frac{\pi}{K}} \theta\right), \\
S_{n}^{y} & =-\left[c_{2} \cos (2 \sqrt{\pi K} \phi)+(-1)^{n} c_{3}\right] \sin \left(\sqrt{\frac{\pi}{K}} \theta\right), \\
S_{n}^{z} & =\sqrt{\frac{\pi}{K}} \partial_{x} \phi+(-1)^{n} c_{1} \cos (2 \sqrt{\pi K} \phi)
\end{aligned}
$$

where $c_{i}$ s are constants as given in Ref. [20]. The Hamiltonian $H_{X Y Z}$ in terms of bosonic fields is the following,

$$
\begin{aligned}
H_{X Y Z}= & H_{0}+a \int \cos \left(2 \sqrt{\frac{\pi}{K}} \theta(x)\right) d x+\Delta \int \cos (4 \sqrt{\pi K} \phi(x)) d x \\
& -h \int \partial_{x} \phi(x) d x
\end{aligned}
$$

One can get the $H_{X Y}$ Hamiltonian by simply putting $\Delta=0$ in the above Hamiltonian. In this derivation, different powers of coefficients $c_{i}$ have been absorbed in the definition of $a, h$ and $\Delta$. The integration of the oscillatory terms in the Hamiltonian yield negligible small ontributions, the origin of the oscillatory terms are the spin operators. So it's a resonably good approximation to keep only the non-oscillatory terms in the Hamiltonian - The Gaussian scaling dimension of these coupling terms, $a, \Delta$ are $1 / K, 4 K$ respectively. The third term $(\Delta)$ of the Hamiltonian tends to order the system into density wave phase, whereas the second term $(a)$ of the Hamiltonian favours the staggered order in the xy plane. Two sine-Gordon couplings terms are from two dual fields. Therefore the model 
Hamiltonian consists of two competing interactions between the ordered phase and the $X Y$ order. This Hamiltonian contains two strongly relevant and mutually nonlocal perturbation over the Gaussian (critical) theory. In such situation strong coupling fixed point is usually determined by the most relevant perturbation whose amplitude grows up according to its Gaussian scaling dimensions and it is not much affected by the less relevant coupling terms. However this is not the general rule, if the two operators exclude each other, i.e., if the field configurations which minimize one perturbation term do not minimize the other. In this case interplay between the two competing relevant operators can produce a novel quantum phase transition through a critical point or a critical line. Therefore we would like to study the RG euation to interpret the quantum phases of the system.

We will now study how the parameters $a, \Delta$ and $K$ flow under RG. The operators in Eq. 17 are related to each other through the operator product expansion; the RG equations for their coefficients will therefore be coupled to each other. Here we derive the RG equations by using perturbative renormalization group approach scheme. We use operator product expansion to derive these RG equations which is independent of boundary condition [24]. RG equations themselves have been established in a perturbative expansion in coupling constant $(g(l))$, they cease to be valid beyond the certain length scale, where $g(l) \sim 1[19]$. The RG equations for the coefficients of Hamiltonian $H_{X Y Z}$ are

$$
\begin{aligned}
\frac{d a}{d l} & =\left(2-\frac{1}{K}\right) a, \\
\frac{d \Delta}{d l} & =(2-4 K) \Delta \\
\frac{d K}{d l} & =\frac{a^{2}}{4}-K^{2} O^{2},
\end{aligned}
$$

These RG equations have trivial $\left(a^{*}=0=\Delta^{*}\right)$ fixed points for any arbitrary $K$. Apart from that these RG equations have also two non-trivial fixed lines, $a=\Delta$ and $a=-\Delta$ for $K=1 / 2$. In our study, there are critical surfaces on which the system flows onto the nontrivial fixed lines $(a= \pm \Delta)$. A density wave states can be characterized when $K \rightarrow 0$ or the staggered ordered when $(K \rightarrow \infty)$. Note that the transition occuring on them are second order. Infinitesimal amount of anisotropy will change the situation drastically, a gapless phase in absence of anisotropy will change to the gapped phase in presence of anisotropy. Since this gapped excitation is not directly related to magnetization, therefore it will not favour to create the plateau phase. When the system is in the plateau phase the transition 
driven by the magnetic field is always of $z=2$ ( $\mathrm{z}$ is the dynamical critical exponent) and thus the plateau shows a square-root behaviour of magnetization. When $a$ is increasing, a second order transition drives system to the in plane XY ordered phase, whose exponents depend on initial couplings and hence are nonuniversal. In absence of planar anisotropy the transition to plateau state is Kosterliz- Thouless-Brezinskii (KTB). When the inplane anisotropy is present, then $z=1$ [21]. Please see Refs. [22, 23] for detail understanding of this subject. A magnetic field larger than the relevant gap of the system drives the system to a gapless phase. This transition is from commensurate phase to incommensurate phase transition.

We have seen the analytical expression of $B_{t o t}$ from Ref. [7] that the total magnetic field increase for the larger values of detuning, therefore larger detuning drives the system from gapped (Mott-insulating) state to gapless superfluid state.

We now discuss, how the effective repulsion will decrease as we increase the detuning between the atomic and laser frequency. It can be explained starting from the JaynesCummings Hamiltonian [25, 26]. Janes-Cummings Hamiltonian for a single atom is

$$
H_{J C}=\omega_{C} a^{\dagger} a+\omega_{0}|e><e|+\lambda\left(a^{\dagger}|g><e|+a|e><g|\right)
$$

$\omega_{C}$ and $\omega_{0}$ are the frequency of the resonant mode of the cavity and of the atomic transition, respectively. $\lambda$ is the Jaynes-Cumming coupling between the cavity mode and the two level system. $a^{\dagger}(a)$ is the creation (annihilation) operator of a photon inside a cavity. $\mid g>$ and $\mid e>$ is respectively the ground state and excited states of the two level system respectively. When we consider large values of photon and atomic frequencies compare to atom-photon coupling $\lambda$, the number of excitations is conserved for this Hamiltonian. Suppose we consider fixed numbers of excitation, $n$. The energy eigenvalues for $n$ excitations is

$$
E_{n}{ }^{ \pm}=n \omega_{C}+\frac{\Delta}{2} \pm \sqrt{n \lambda^{2}+\frac{\Delta^{2}}{4}}
$$

Here $\Delta=\left(\omega_{0}-\omega_{c}\right)$ and $n \geq 1$. Now we consider an array of cavities, the basic Hamiltonian for each cavity is the same as that of Eq.19. Here we consider the system with one excitation of energy $E_{1}$ in each cavity and the lowest energy of two excitation in each cavity is $E_{2}$. Therefore to create one additional excitation in each cavity requires energy

$$
E_{2}-2 E_{1}=2 \sqrt{\lambda^{2}+\frac{\Delta^{2}}{4}}-\sqrt{2 \lambda^{2}+\frac{\Delta^{2}}{4}}-\frac{\Delta}{2} .
$$


Which one may consider as an effective on-site repulsion because it measures the difference between the energy of two and single excitation (polariton in each cavity). This effective repulsion decreases as we increase the detune factor. Therefore we can conclude that for $\Delta=0$, double occupation never occurs, indicating a Mott insulating behaviour. When $\Delta$ is much larger than the coupling $\lambda$ then the occupation number larger than one occurs as to be expected for a photonic superfluid regime. In our quantum field theoretical calculations, we have also predicted that the large detuning drives the system from gapped Mott insulating phase to the gapless superfluid phase.

\section{CONCLUSIONS}

At first we have done the quantum field theoretical analysis of an effective spin model in coupled optical cavity arrays. We have predicted two quantum phases, Mott insulator and photonic superfludity. Anisotropy in the exchange interaction has also created a gapped phase. An infinite order KTB transition has been replaced by the second order XY transition. The rigorous quantum field theoretical derivation of this manuscript is absent in all previous studies and also we provide physical explanation of the transition process based on JaynesCummings Hamiltonian.

\section{Acknowledgments}

The author would like to acknowledge The Center for Condensed Matter Theory of the Physics Department of IISc for providing working space and also Dr. R. C. Sarasij for reading the manuscript very critically.

[1] C. N. R. Rao and T. V. Ramakrishnan in Superconductivity Today (Universities Press, Hyderabad, 1999).

[2] K. K. Likharev in Dynamics of Josephson junction and circuits (Gordon and Breach 1988).

[3] J. Hecker. Denschlog et al., J. Phys. B: At. Mol. Opt Phys, 35, 3095 (2002).

[4] D. Jaksch and P. Zoller, Annals of Physics 315, 52 (2005).

[5] A. D. Greentree et al., Nature Phys. 466, 856 (2006). 


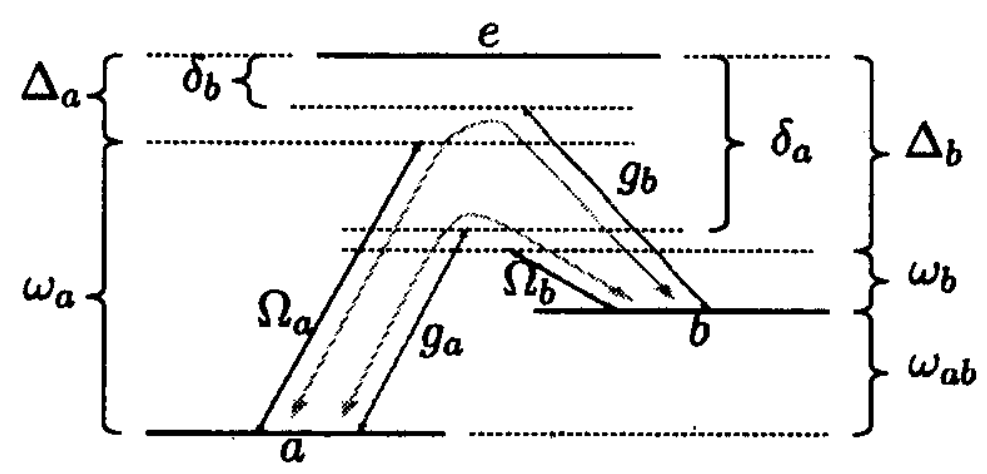

FIG. 1: Level structure, driving laserrs, and relevant couplings to the cavity mode to generate effective $\sigma^{x} \sigma^{x}$ and $\sigma^{y} \sigma^{y}$ couplings for one atom. The cavity mode couples with strength $g_{a}$ and $g_{b}$ to transition $|a>\leftrightarrow| e>$ and $|b>\leftrightarrow| e>$ respectively. One laser frequency $\omega_{a}$ couples to the transition $|a>\leftrightarrow| e>$ with Rabi frequency $\Omega_{a}$ and another with frequency $\omega_{b}$ to $|b>\leftrightarrow| e>$ with $\Omega_{b}$. The dominant two-photon processes are indicated in faint arrows. Reprinted with permission from American Physical Society. Analytical expressions and physical meaning of different symbols have given in Ref. [7].

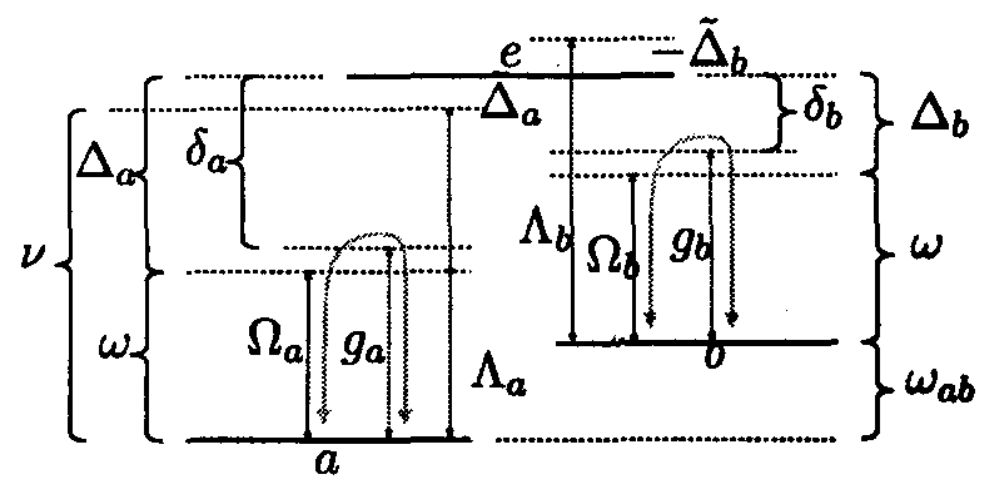

FIG. 2: Level structure, driving laserrs, and relevant couplings to the cavity mode to generate effective $\sigma^{z} \sigma^{z}$ couplings for one atom. The cavity mode couples with strength $g_{a}$ and $g_{b}$ to transition $|a>\leftrightarrow| e>$ and $|b>\leftrightarrow| e>$ respectively. Two lasers with frequencies $\omega$ and $\nu$ couple with Rabi frequencies $\Omega_{a}$, respectively, $\Lambda_{a}$ to transition $|a>\leftrightarrow| e>$ and $\Omega_{b}$, respectively, $\Lambda_{b}$ to transition $|b>\leftrightarrow| e>$. The dominant two-photon processes are indicated in faint arrows. Reprinted with permission from American Physical Society. Analytical expressions and physical meaning of different symbols have given in Ref.[7]. 
[6] Michael J. Hartmann, Fernando G. S. L Brando and Martin B. Plenio, Nature Phys. 462, 849 (2006); Michael J. Hartmann, Fernando G. S. L Brando and Martin B. Plenio, Laser and Photonics Rev. 2, 527 (2008).

[7] Michael J. Hartmann, Fernando G. S. L Brando and Martin B. Plenio, Phys. Rev. Lett 99, 160501 (2007).

[8] A-C.Ji, X. C. Xie, and W. M. Liu, Phys. Rev.Lett. 99, 183602 (2007).

[9] T. Byrnes, N. Y. Kim, K. Kusudo, and Y. Yamamoto, Phys. Rev. B 78, 075320 (2008).

[10] I. Carusotto et al., arXiv:0812.4195 (2008).

[11] M. J. Bhaseen, M. Hohenadler, A. O. Silver, and B. D. Simons, Phys. Rev. Lett. 102

[12] A. Tomadin et al. arXiv:0904.4437 (2009).

[13] J. Zhao, A. W. Sandvik, and K. Ueda, arXiv:0806.3603 (2008).

[14] P. Pippan, H. G. Evertz, and M. Hohenadler, arXiv: 0904.1350 (2009).

[15] D. Rossini, and R. Fazio, Phys. Rev. Lett. 99, 186401 (2007).

[16] M. Aichhorn et al., Phys. Rev. Lett. 100, 216401 (2008).

[17] S. Schmidt, and G. Blatter, arXiv:0905.3344.

[18] D. G. Angelakis, M. F. Santos and S. Bose, Phys. Rev. A 76, R031805 (2007).

[19] T. Giamarchi in Quantum Physics in One Dimension (Clarendon Press, Oxford 2004).

[20] S. Lukyanov and A. Zamolodchikov, Nucl. Phys. B 493, 571 (1997).

[21] In quantum phase transitions, in addition to the standard critical exponent it is useful to define an additional exponent $\mathrm{z}$, called the dynamical exponent, which tells us how a characteristic length in the time direction is related to a length in the spatial direction $\xi_{\tau} \sim \xi_{x}{ }^{z}$. For quantum problem time plays a special role and this special direction has no reasons to have the same exponent as the spatial one. Deep inside the Mott insulating phase, particle and hole excitations are gapped. The system is almost alike to the atomic limit in the deep Mott insulating phase. When one approaches the phase boundary from the deep Mott insulating phase then the dispersion relation is quadratic $\omega \sim k^{2}(z=2)$. But the situation is different at the end of CDW phase and the starting point of XY staggered order phase. At around the multical critical point $z$ is 1 . Please see the Refs. [22, 23] and for a detailed understanding of this subject.

[22] Subir Sachdev in "Quantum Phase Transition" (Cambridge University Press Cambridge, 1998). 
[23] S. L. Sondhi, et al. Rev. Mod. Phys. 69, 315 (1997).

[24] J. Cardy in Scaling and Renormalization in Statistical Physics (Cambridge University Press, Cambridge 1996); I. Affleck in Fields, Strings and and Critical Phenomena, ed E. Brezin and J. Zinn-Justin (North-Holland, Amstardam 1989).

[25] Jaynes, E. T. and F. W. Cummings, Proc IEEE, 51, 89 (1963).

[26] S. Horoche and J. M. Raimond in Exploring the Quantum Atoms, Cavities, and Photons, (Oxford University Press, 2006). 